\title{
Comparative Study between Intralesional Injection versus Intradermal Injection of Candida Albicans Antigen as An Effective Treatment of Common Warts
}

Abd El-Shakour Abd El- Hafiz Al Mohammady, Hassan Mamdoh Abd El-Aziz, Loay Hazem Aamer

Department of Dermatology, Venerology and Andrology; Faculty of Medicine, Al-Azhar University

*Corresponding author: Loay Hazem Aamer; Mobile: (+20)01099226613; E-mail: loaibeck@gmail.com

\begin{abstract}
Background: Warts, or verrucae, are benign epithelial proliferations of skin and mucosa caused by infection with human papilloma virus (HPV). They are common skin condition that can range in severity from a minor nuisance that resolves spontaneously to troublesome, chronic condition.

Objective: The aim of the present study is to compare between intralesional injection of candida albicans antigen versus intradermal injection as an effective treatment of common warts.

Patients and methods: This study included 60 (sixty) patients (adult males and females) were suffering from common warts. The patients were divided into two equal groups of 30 patients each: Group A treated by intradermal injection of candida albicans antigen. Group $\mathbf{B}$ treated by intralesional injection of candida albicans antigen in one of their warts (mother wart) which was chosen and marked. The patients were collected from the outpatient's clinics of Dermatology and Venereology Department of Al- Hossein University Hospital.

Results: As we compared the response to candida albicans antigen injection, we found a highly significant cure response in intralesional injections over intradermal injections. After 6 months follow up in patients who responded by complete cure (29 patients), there was no recurrence in 26 patients $(89.6 \%)$ and only 3 cases $(10.4 \%)$ developed partially recurrence. As regard to side effects there were no side effects in 43 cases (71.7\%). Conclusion: candida albicans antigen injection seems to be promising effective and safe remedy for cutaneous warts with good cure rates and excellent safety profile.
\end{abstract}

Keyword: Intralesional injection, Intradermal injection of candida albicans antigen, Warts

\section{INTRODUCTION}

Warts are benign lesions that occur in the mucosa and skin ${ }^{(\mathbf{1})}$. These are a common medical problem, especially in Whites ${ }^{(2)}$.

Warts are common dermatological skin disease that affects $7-10 \%$ of the general population. Warts constitute the commonest cutaneous manifestation of human papilloma virus (HPV) that infects epithelial tissues of skin and mucous membranes. There are over 150 distinct HPV subtypes; some tend to infect specific body sites and produce characteristic proliferative lesions at those sites. Many types of warts have been identified: common wart, flat warts, plantar warts and genital warts ${ }^{(3)}$.

Different modalities

cutaneous warts are available. Local treatments include salicylic acid, intralesional interferons (alpha, beta, and gamma), dinitrochlorobenzene, intralesional bleomycin and intralesional 5fluorouracil. Other treatments include photodynamic therapy, cautery, laser, immunotherapy and cryotherapy ${ }^{(3)}$. Antigens used for intralesional immunotherapy include tuberculin (PPD), candida, trichophyton; and mumps, measles, and rubella (MMR) ${ }^{(4)}$.

Candida antigen is made from the culture filtrate and cells of two strains of Candida albicans. It's indicated in origin for use as a recall antigen for detecting delayed-type hypersensitivity by intracutaneous (intradermal) testing. The inflammatory response associated with the delayed-type hypersensitivity (DTH) reaction is characterized by an infiltration of lymphocytes and macrophages at the site of antigen deposition. Specific cell types that appear to play a major role in the DTH response include CD4 and CD8 T lymphocytes (5). Intralesional immunotherapy utilizes the ability of the immune system to mount a delayed type hypersensitivity response to various antigens and also the wart tissue. This therapy was found to be associated with the production of Th1 cytokines which activate cytotoxic and natural killer cells to eradicate HPV infection. This clears not only the local warts but also distant warts unlike traditional wart therapies ${ }^{(6)}$.

\section{AIM OF THE WORK}

The aim of the present study is to compare between intralesional injection of candida albicans antigen versus intradermal injection as an effective treatment of common warts.

\section{PATIENTS AND METHODS}

- This study included 60 (sixty) patients (adult males and females) who were suffering from common warts.

- The patients were divided into two equal groups of 30 patients each :

1. Group A treated by intradermal injection of candida albicans antigen.

2. Group B treated by intralesional injection of candida albicans antigen in one of their warts (mother wart), which was chosen and marked. 
- The patients collected from the outpatient's clinics of Dermatology and Venereology Department of Al- Hossein University Hospital.

\section{For each patient the following were done:}

Complete history taking.

General and dermatological examination.

Identify clinical type of warts and site of lesions.

$\checkmark$ Take photos for the lesions at first visit and follow up visits.

\section{Patients}

\section{Inclusion criteria}

Patients chosen were:

1- Healthy individuals of both sex

2- Age group 18-55

3- With a history of more than six months common warts.

\section{Exclusion criteria}

1. Immuno-compromised patients

2. Those who were taking immunosuppressant medication due to health reasons

3. Patients with compromised wound healing, poor tissue viability, diabetes mellitus, peripheral neuropathy and peripheral arterial or venous disease

4. Pregnant or breastfeeding.

5. Patients who had absolute contraindication for local anesthesia, lack of consent or noncooperation.

There are systemic or local adverse reactions found during or after the study including:

a- Pain during and after treatment.

b- Erythema.

c- Swelling.

d- Itching and skin rash.

-The measures used to minimize these risks were complete history taking, assurance, complete aseptic technique and use of local anesthetic solution as lignocaine.

-Any unexpected complication that appeared during the course of the research was cleared to the participants and the ethical committee on time.

-Every participant had had a code number, photos taken to the diseased part only.

-All the records were confidential.

\section{Methods}

All the patients subjected to the following:

1. Complete history taking.

2. Thorough general and dermatological clinical examinations.

3. Examination and evaluation of warts.

4. Written informed consent from every patient obtained before the procedure. An approval of the study was obtained from Al-Azhar University academic and ethical committee.
5. Photographs were took before starting therapy and at every visit to support the recorded data and at the end of the therapy.

6. Group A patients were injected with purified candida albicans antigen intradermally.

- Injections were given intradermally using a 27-guage needle, and a total of four injections were administered at 3-week intervals. The volume of the test antigen used was $0.1 \mathrm{ml}$ on every occasion.

7. Group B patients were injected with purified candida albicans antigen intralesionally.

Injections were given into the substance of the wart using a 27-guage needle, and a total of four injections were administered at 3-week intervals. The same wart was injected on all the three occasions in every patient. The patients were examined at each follow-up injection and any regression in the size of the warts was noted down The volume of the test antigen used was $0.1 \mathrm{ml}$ on every occasion irrespective of the severity of response to the initial intradermal test dose. Thus, a total of $0.3 \mathrm{ml}$ of the test antigen was used in each patient. The patients were examined at each follow-up injection and any regression in the size of the warts was noted down

8. In each group an average of 3 sessions every 3 weeks performed.

9. Follow up for 8 weeks after the end of the last treatment was done to asses any recurrence or side effects.

10. The duration of the study was six months.

The study was approved by the Ethics Board of Al-Azhar University.

\section{Statistical methods}

Statistical presentation and analysis of the present study was conducted, using the mean, standard deviation, or the frequency and percentage. Unpaired student t-test was used to compare between the two groups in quantitative data and chi-square test was used to compare the qualitative data by SPSS V20. Fisher's exact test and Yates' corrected chi-square were computed when appropriate.

Significant level: Non-significant $>0.05$, significant $<0.05^{*}$, and highly significant $<0.001 * *$

\section{RESULTS}

Baseline characters of the studied patients in both groups are presented in tables (1,2, and 3). There was no statistically significant difference between the two groups in the demographic data (age, sex and race) and the clinical parameters. 
Table (1) Age of patients and duration of warts

\begin{tabular}{|c|c|c|}
\hline & Range & Mean \pm SD \\
\hline Age & $20-54$ & $34.08 \pm 7.85$ \\
\hline Duration of warts & $6-12(\mathrm{~m})$ & $6.92 \pm 1.48$ \\
\hline
\end{tabular}

Table (2) Mode of injection

\begin{tabular}{|c|c|c|}
\hline Type of injections & $\mathrm{N}$ & $\%$ \\
\hline Intradermal & 30 & 50.0 \\
\hline Intralesional & 30 & 50.0 \\
\hline Total & 60 & 100.0 \\
\hline
\end{tabular}

Table (3) Relation between type of injections and sex in patients

\begin{tabular}{|c|c|c|c|c|c|c|}
\hline \multirow{2}{*}{ Sex } & \multicolumn{7}{|c|}{ Type of injections } \\
\cline { 3 - 8 } & \multicolumn{2}{|c|}{ Intradermal } & \multicolumn{2}{c|}{ Intralesional } & \multicolumn{2}{c|}{ Total } \\
\cline { 3 - 8 } & $\mathrm{N}$ & $\%$ & $\mathrm{~N}$ & $\%$ & $\mathrm{~N}$ & $\%$ \\
\hline Female & 14 & 46.7 & 19 & 63.3 & 33 & 55.0 \\
\hline Male & 16 & 53.3 & 11 & 36.7 & 27 & 45.0 \\
\hline Total & 30 & 100.0 & 30 & 100.0 & 60 & 100.0 \\
\hline \multirow{2}{*}{ Chi-square } & $\mathrm{X}^{2}$ & \multicolumn{7}{|c|}{1.684} \\
\cline { 2 - 8 } & P-value & \multicolumn{7}{c|}{0.194} \\
\hline
\end{tabular}

Table (3) shows non-statistical significant difference between type of injections and sex in patients, $\mathrm{P}>0.05$

Table (4) Relation between type of injections and number of warts in patients

\begin{tabular}{|c|c|c|c|c|c|c|c|}
\hline \multirow{3}{*}{\multicolumn{2}{|c|}{ number of warts }} & \multicolumn{6}{|c|}{ Type of injections } \\
\hline & & \multicolumn{2}{|c|}{ Intradermal } & \multicolumn{2}{|c|}{ Intralesional } & \multicolumn{2}{|c|}{ Total } \\
\hline & & $\mathrm{N}$ & $\%$ & $\mathrm{~N}$ & $\%$ & $\mathrm{~N}$ & $\%$ \\
\hline \multicolumn{2}{|c|}{ Single } & 15 & 50.0 & 18 & 60.0 & 33 & 55.0 \\
\hline \multicolumn{2}{|c|}{ Two } & 5 & 16.7 & 2 & 6.7 & 7 & 11.7 \\
\hline \multicolumn{2}{|c|}{ Multiple } & 10 & 33.3 & 10 & 33.3 & 20 & 33.3 \\
\hline \multicolumn{2}{|c|}{ Total } & 30 & 100.0 & 30 & 100.0 & 60 & 100.0 \\
\hline \multirow{2}{*}{ Chi-square } & $x^{2}$ & \multicolumn{6}{|c|}{1.558} \\
\hline & $\mathrm{P}$-value & \multicolumn{6}{|c|}{0.459} \\
\hline
\end{tabular}

Table (4) shows non statistical significant difference between type of injections and number of warts in patients, $\mathrm{P}>0.05$

Table (5) Relation between type of injections and type of wart in patients

\begin{tabular}{|c|c|c|c|c|c|c|c|}
\hline \multirow{3}{*}{\multicolumn{2}{|c|}{ type of wart }} & \multicolumn{6}{|c|}{ Type of injections } \\
\hline & & \multicolumn{2}{|c|}{ Intradermal } & \multicolumn{2}{|c|}{ Intralesional } & \multicolumn{2}{|c|}{ Total } \\
\hline & & $\mathrm{N}$ & $\%$ & $\mathrm{~N}$ & $\%$ & $\mathrm{~N}$ & $\%$ \\
\hline \multicolumn{2}{|c|}{ Common wart } & 28 & 93.3 & 24 & 80.0 & 52 & 86.7 \\
\hline \multicolumn{2}{|c|}{ Plane wart } & 2 & 6.7 & 5 & 16.7 & 7 & 11.7 \\
\hline \multicolumn{2}{|c|}{ Both } & 0 & 0.0 & 1 & 3.3 & 1 & 1.7 \\
\hline \multicolumn{2}{|c|}{ Total } & 30 & 100.0 & 30 & 100.0 & 60 & 100.0 \\
\hline \multirow{2}{*}{ Chi-square } & $x^{2}$ & \multicolumn{6}{|c|}{2.593} \\
\hline & P-value & \multicolumn{6}{|c|}{0.273} \\
\hline
\end{tabular}

Table (5) shows non statistical significant difference between type of injections and type of wart in patients, $\mathrm{P}>0.05$.

Table (6) Relation between Type of injections and clinical response in patients

\begin{tabular}{|c|c|c|c|c|c|c|c|}
\hline \multirow{3}{*}{\multicolumn{2}{|c|}{ clinical response }} & \multicolumn{6}{|c|}{ Type of injections } \\
\hline & & \multicolumn{2}{|c|}{ Intradermal } & \multicolumn{2}{|c|}{ Intralesional } & \multicolumn{2}{|c|}{ Total } \\
\hline & & $\mathrm{N}$ & $\%$ & $\mathrm{~N}$ & $\%$ & $\mathrm{~N}$ & $\%$ \\
\hline \multicolumn{2}{|c|}{ Complete cure } & 5 & 16.7 & 24 & 80.0 & 29 & 48.3 \\
\hline \multicolumn{2}{|c|}{ Partial cure } & 5 & 16.7 & 2 & 6.7 & 7 & 11.7 \\
\hline \multicolumn{2}{|c|}{ No response } & 20 & 66.7 & 4 & 13.3 & 24 & 40.0 \\
\hline \multicolumn{2}{|c|}{ Total } & 30 & 100.0 & 30 & 100.0 & 60 & 100.0 \\
\hline \multirow{2}{*}{ Chi-square } & $x^{2}$ & \multicolumn{6}{|c|}{24.401} \\
\hline & P-value & \multicolumn{6}{|c|}{$<0.001 * *$} \\
\hline
\end{tabular}

Table (6) shows statistical significant difference between type of injections and clinical response in patients, $\mathrm{P}<0.001 * *$. Patients responded to intralesional injection better than intradermal injection of purified candida albicans antigen. 
Table (7) Relation between type of injections and recurrence after 6 months follow up in completely improved patients

\begin{tabular}{|c|c|c|c|c|c|c|c|}
\hline \multirow{3}{*}{\multicolumn{2}{|c|}{6 months follow up }} & \multicolumn{6}{|c|}{ Type of injections } \\
\hline & & \multicolumn{2}{|c|}{ Intradermal } & \multicolumn{2}{|c|}{ Intralesional } & \multicolumn{2}{|c|}{ Total } \\
\hline & & $\mathrm{N}$ & $\%$ & $\mathrm{~N}$ & $\%$ & $\mathrm{~N}$ & $\%$ \\
\hline \multicolumn{2}{|c|}{ No recurrence } & 5 & 83.3 & 21 & 87.5 & 26 & 86.7 \\
\hline \multicolumn{2}{|c|}{ Partially recurrence } & 1 & 16.7 & 3 & 12.5 & 4 & 13.3 \\
\hline \multicolumn{2}{|c|}{ Total } & 6 & 100.0 & 24 & 100.0 & 30 & 100.0 \\
\hline \multirow{2}{*}{ Chi-square } & $x^{2}$ & \multicolumn{6}{|c|}{0.072} \\
\hline & P-value & \multicolumn{6}{|c|}{0.788} \\
\hline
\end{tabular}

Table (7) shows non statistical significant difference between type of injections and recurrence after 6 months follow up in patients, $\mathrm{P}>0.05$.

Table (8) Relation between type of injections and side effects in patients

\begin{tabular}{|c|c|c|c|c|c|c|}
\hline \multirow{2}{*}{ side effects } & \multicolumn{7}{|c|}{ Type of injections } \\
\cline { 2 - 7 } & Intradermal & \multicolumn{1}{|c|}{ Intralesional } & \multicolumn{2}{c|}{ Chi-square } \\
\cline { 2 - 7 } & $\mathrm{N}$ & $\%$ & $\mathrm{~N}$ & $\%$ & $\mathrm{X}^{2}$ & P-value \\
\hline No & 23 & 76.6 & 9 & 30 & 13.125 & $<0.001^{* *}$ \\
\hline Pain & 4 & 13 & 12 & 40 & 5.455 & $0.020^{*}$ \\
\hline Erythema & 1 & 3 & 4 & 13 & 1.964 & 0.161 \\
\hline Swelling & 0 & 0 & 1 & 3 & 1.017 & 0.313 \\
\hline Itching & 2 & 6 & 4 & 13 & 0.741 & 0.389 \\
\hline Total side effects & 7 & 23.3 & 21 & 70 & 13.125 & $<0.001^{* *}$ \\
\hline Total & 30 & 100.0 & 30 & 100.0 & & \\
\hline
\end{tabular}

Table (8) shows high statistical significant difference between type of injections and side effects in patients, $P>0.001$. Pain as side effect was more in intralesional injection than intradermal as shown.

Table (9) Effects of intralesional injection of mother wart (biggest one) on the other one/s in cured patients

\begin{tabular}{|c|c|c|}
\hline $\begin{array}{c}\text { Response of distant } \\
\text { wart/s when inject mother one }\end{array}$ & $\begin{array}{c}\text { 9 multiple cured } \\
\text { with intralesional injection }\end{array}$ & $\mathbf{1 0 0 \%}$ \\
\hline Resolution & 7 & $\mathbf{7 8 \%}$ \\
\hline No response & 2 & $\mathbf{2 2 \%}$ \\
\hline
\end{tabular}

\section{DISCUSSION}

Cutaneous warts represent a troublesome therapeutic issue for both patients and physicians. Despite the existence of numerous therapeutic modalities, treatment of warts still represents a real challenge and a uniformly effective remedy has not been explored to date ${ }^{(7)}$. Many destructive and immunotherapeutic modalities were used to treat different types of cutaneous warts. Destructive therapies include medical agents, such as podophyllotoxin and trichloroacetic acid in high concentration, and surgical methods such as aggressive cryosurgery, curettage, surgical excision, electrocautery, laser ablation, and other as photodynamic therapy and intralesional immunotherapy ${ }^{(\mathbf{8})}$. Medical agents as podophyllin, podophyllotoxin, trichloroacetic acid, sinecatechins, bleomycin and fluorouracil have been tried, however most of them are largely unsatisfactory and none of them offers a guarantee of cure and recurrence is common ${ }^{(4)}$.

Immunotherapy is a promising modality for recurrent and/or resistant warts which could lead to resolution without any physical changes or scarring, and in addition would augment the host response against the causative agent, thereby leading to complete resolution and decreased recurrences ${ }^{\left({ }^{9}\right)}$.

Regarding verruca vulgaris: candida immunotherapy seems to be an alternative treatment option in patients with whom the warts are either resistant or not responding to destructive modes of treatment ${ }^{(\mathbf{1 0})}$. As regard our results with Candida antigen treatment comparing between intradermal and intralesional injections, no statistical significance was found as regard to type 
of injections and sex or age in patients, $\mathrm{p}$-value was 0.194 ( >0.05). As regard our results with Candida antigen treatment comparing between intradermal and intralesional injections, no statistical significance was found as regard to type of injections and number of injections used in patients, $\mathrm{p}$-value was $>0.05$.

A better response might have been obtained if the volume of Candida antigen injected was increased, if more than a wart (not only the target wart) were treated at a time, or if more treatment sessions were used as in Gupta (11) showing $88.9 \%$ cure rate with 10 injections as an emerging therapy, different protocols should be tried in an attempt to improve the response rates.

As regard our results with Candida antigen treatment comparing between intradermal and intralesional injections, a statistical significance was found with regard to type of injections and clinical response in patients, p-value was $<0.001$.

Regarding patients who received intradermal injections, there was complete cure in 5 patients $(16.7 \%)$, partial cure in 5 patients (16.7\%) and no response in 20 patients $(66.7 \%)$.

As regard patients who received intralesional injections, there was complete cure in 24 patients $(80 \%)$, partial cure in 2 patients $(6.7 \%)$ and no response in 4 patients $(13.3 \%)$.

This is lower than that reported by Kim $\boldsymbol{e t}$ al. ${ }^{(6)}(82 \%)$. As we compared the response to candida albicans antigen injection, we found a highly significant cure response in intralesional injections over intradermal injections $\mathrm{p}$ value $<0.001$.

The differences in the study population selected for treatment, the number of the studied patients, the sensitivity degree to the injected antigen, types of candida antigen used, injection plan, and the number, type, duration and resistance of warts may be responsible for the difference between the results of our study and other related studies utilizing Candida antigens.

The clearance of untreated warts, including the nearby and distant lesions, was also reported by other studies utilizing intralesional antigen injection for the treatment of different types of warts: Gupta ${ }^{(11)}$ (killed mycobacterium vaccine, 89\%); Nofal $\boldsymbol{e t}$ al. ${ }^{(7)}$ (MMR vaccine, 74.5\%) and Na et al. ${ }^{(12)}$ (MMR vaccine, 46.7\%).

This strongly indicates the development of a widespread cell-mediated immunity against HPV as a response to antigen injection; an observation that represents a highly promising advantage of intralesional antigen immunotherapy over traditional therapies ${ }^{(7)}$.

\section{CONCLUSION AND RECOMMENDATIONS}

In conclusion, intralesional candida albicans antigen seems to be promising effective and safe remedy for cutaneous warts with good cure rates and excellent safety profile. It's inexpensive and has the potential advantages of widespread and sustained effects against HPV. We recommend the widespread use and more sessions of intralesional candida albicans in the treatment of different types of cutaneous warts, at different age groups, on a much larger scale and in comparison with other traditional therapeutic modalities to accurately define their place in the challenging field of wart therapy.

\section{REFERENCES}

1. Khozeimeh F, Jabbari F, Mahboubi Y et al. (2017): Intralesional immunotherapy compared to cryotherapy in the treatment of warts. Int J Dermatol., 56(4):474-478.

2. Augustin M, Kirsten N, Körber A et al. (2019): Prevalence, predictors and comorbidity of dry skin in the general population. J Eur Acad Dermatol Venereol., 33(1):147-150.

3. Sterling JC, Gibbs S and Haque SS (2014): Management of cutaneous warts. Br J Dermatol., 171(4):696-712.

4. Johnson SM, Helm RM, Roberson PK et al. (2005): Intralesional immunotherapy of warts with mumps, Candida, and Trichophyton skin test antigens: a single-blinded, randomized, and controlled trial. Arch Dermatol., 141(5):589-94.

5. Paul WE (1993): Fundamental Immunology, 3rd Ed., pp 75-76, Raven Press, New York, https://www.ncbi.nlm.nih.gov/nlmcatalog/9303809.

6. Kim K, Horn T, Pharis J et al. (2010): Phase 1 clinical trial of intralesional injection of Candida antigen for the treatment of warts. Arch Dermatol., 146:1431-1433.

7. Nofal A, Nofal E, Yosef A et al. (2015): Treatment of recalcitrant warts with intralesional measles, mumps, and rubella vaccine: a promising approach. Int J Dermatol., 54(6):667-71.

8. Leung L (2011): Recalcitrant nongenital warts. Aust Fam Physician, 40(1-2):40-2.

9. Sinha S, Relhan V and Garg VK (2015): Immunomodulators in warts: Unexplored or ineffective? Ind. J. Dermatol., 60: 118-122.

10. Gearhart A, Randall T, Buckley $\mathrm{R}$ et al. (2011): Human papillomavirus (HPV). http://www.emedicine.com/med/topic219110.htm

11. Gupta R (2011): Plantar warts treated with topical adapalene. Indian J Dermatol., 56:513-516.

12. Na CH, Choi H, Song SH et al. (2014): Two-year experience of using the measles, mumps and rubella vaccine as intralesional immunotherapy for warts. Clin Exp Dermatol., 39 (5):583-9. 